\title{
LETTER
}

Lymphoma

\section{Identification of the SRC-family tyrosine kinase HCK as a therapeutic target in mantle cell lymphoma}

\author{
Hildo C. Lantermans ${ }^{1,2} \cdot$ Marthe Minderman $^{1,2} \cdot$ Annemieke Kuil $^{1,2} \cdot$ Marie-José Kersten $^{2,3} \cdot$ Steven T. Pals $^{1,2}$. \\ Marcel Spaargaren $\mathbb{D}^{1,2}$
}

Received: 25 February 2020 / Revised: 18 May 2020 / Accepted: 16 June 2020 / Published online: 26 June 2020

(c) The Author(s) 2020. This article is published with open access

\begin{abstract}
Mantle cell lymphoma (MCL) is an aggressive non-Hodgkin lymphoma subtype arising from naïve B cells. Although novel therapeutics have improved patient prognosis, drug resistance remains a key problem. Here, we show that the SRC-family tyrosine kinase hematopoietic cell kinase (HCK), which is primarily expressed in the hematopoietic lineage but not in mature $\mathrm{B}$ cells, is aberrantly expressed in MCL, and that high expression of HCK is associated with inferior prognosis of MCL patients. HCK expression is controlled by the toll-like receptor (TLR) adaptor protein MYD88 and can be enhanced by TLR agonists in MCL cell lines and primary MCL. In line with this, primary MCL with high HCK expression are enriched for a TLR-signaling pathway gene set. Silencing of HCK expression results in cell cycle arrest and apoptosis. Furthermore, HCK controls integrin-mediated adhesion of MCL cells to extracellular matrix and stromal cells. Taken together, our data indicate that TLR/MYD88-controlled aberrant expression of HCK plays a critical role in MCL proliferation and survival as well as in retention of the malignant cells in the growth- and survival-supporting lymphoid organ microenvironment, thereby contributing to lymphomagenesis. These novel insights provide a strong rationale for therapeutic targeting of HCK in MCL.
\end{abstract}

\section{Introduction}

Mantle cell lymphoma (MCL) is an aggressive lymphoma subtype with poor clinical outcome, characterized by the $\mathrm{t}(11 ; 14)(\mathrm{q} 13 ; \mathrm{q} 32)$ translocation, resulting in overexpression of Cyclin D1. Advances in MCL therapy have improved patient prognosis, but due to primary and secondary resistance there is a high clinical need for novel therapeutic targets [1].

These authors contributed equally: Steven T. Pals, Marcel Spaargaren

Supplementary information The online version of this article (https:// doi.org/10.1038/s41375-020-0934-6) contains supplementary material, which is available to authorized users.

Marcel Spaargaren

marcel.spaargaren@amsterdamumc.nl

1 Department of Pathology, Cancer Center Amsterdam, Amsterdam UMC, University of Amsterdam, Amsterdam, The Netherlands

2 Lymphoma and Myeloma Center Amsterdam - LYMMCARE, Amsterdam, The Netherlands

3 Department of Hematology, Cancer Center Amsterdam, Amsterdam UMC, University of Amsterdam, Amsterdam, The Netherlands
The SRC-family tyrosine kinase hematopoietic cell kinase $(\mathrm{HCK})$, which is predominantly expressed in the hematopoietic lineage, has been implicated in various cellular processes including chemokine signaling, proliferation, apoptosis, and immune cell activation [2-5]. Furthermore, HCK has been shown to be deregulated in hematological and solid malignancies, and plays a role in tumor cell survival [6-8]. In Waldenström's Macroglobulinemia (WM) and activated B-cell type diffuse large B-cell lymphoma (ABC-DLBCL) cells harboring the pathogenic MYD88-L265P mutation, mutant MYD88dependent HCK expression was observed and HCK knockdown reduces cell viability, implying that HCK targeting could be beneficial in patients with lymphomas with mutant MYD88 [8]. Although no MYD88 mutations have been reported in MCL, MYD88-regulatory toll-like receptors (TLRs) are upregulated and TLR stimulation supports cell proliferation and survival [9-11]. Here, we show that HCK is aberrantly expressed in MCL in a TLR- and MYD88-dependent fashion and that high HCK expression correlates with poor prognosis. Furthermore, HCK controls proliferation and survival as well as integrin-mediated adhesion of MCL cells. Our results indicate that HCK inhibition has therapeutic potential in MCL, and 
possibly also in other lymphomas that do not harbor MYD88 mutations.

\section{Methods}

For information about shRNA cloning, cell-culture, transductions, stimulations, immunoblotting, primary cell isolation, RT-qPCR, cell-cycle analysis, Annexin-V staining, integrin staining, and Gene Set Enrichment Analysis (GSEA) see Supplementary "Materials and Methods".

For adhesion experiments, JeKo-1 cells expressing inducible shRNA's were pre-treated with doxycycline and allowed to adhere to 96-well-plates, coated with fibronectin or the stromal cell line HS-27a expressing GFP, in the absence/presence of phorbol-12-myristate 13-acetate (PMA). To quantify adhesion to HS-27a, cells were trypsinized and quantified by flow cytometric analysis. As indicated, JeKo-1 or primary MCL cells were treated for $30 \mathrm{~min}$ at $37^{\circ} \mathrm{C}$ (A419259) or $4{ }^{\circ} \mathrm{C}$ (HP2/1, TS1/22) prior to allowing cells to adhere to fibronectin-coated plates or the stromal cell line HS-27a-GFP, For further details see Supplementary "Materials and Methods".

\section{Results and discussion}

To evaluate $H C K$ mRNA expression across various lymphoid malignancies, we analyzed publicly available microarray data. In germinal center B-cells and normal plasma cells $H C K$ was below the detection-threshold, whereas it was weakly expressed in memory B-cells (Fig. 1a). In tumor biopsies of ABC- and Germinal Center (GC)DLBCL $H C K$ was expressed, with higher levels of HCK in ABC-DLBCL (Fig. 1a). Expression of $H C K$ in MCL was comparable to GC-DLBCL and higher than in WM, multiple myeloma (MM), or chronic lymphocytic leukemia samples (Fig. 1a). Importantly, high $H C K$ expression was found to correlate with poor overall survival in MCL patients. The median overall survival was 17 months in HCK-high vs. 40 months in HCK-low patients and the 5 year survival was $14 \%$ in HCK-high vs. $41 \%$ in HCK-low patients (Fig. 1b).

Next, we analyzed HCK protein expression in a panel of WM, DLBCL and MCL cell lines. In the MCL cell lines Maver-1, Granta-519, JeKo-1, Rec-1, and Mino HCK levels were similar to ABC-DLBCLs without MYD88 mutations, whereas in Z-138 HCK was barely detectable (Fig. 1c). Notably, Z-138 represents an atypical MCL cell line, with blastoid transformation in the terminal phase of disease. In GC-DLBCL cell lines HCK was hardly detectable, whereas it was expressed in all ABC-DLBCL cell lines independent of the presence of a MYD88 mutation, although the levels were higher when MYD88 was mutated (Fig. 1c). Notably, the WM cell lines, expressing mutant MYD88, contained less HCK than most MCL lines (Fig. 1c). Higher expression of HCK was observed in the WM cell line MWCL-1 than in BCWM.1, in accordance with a previous study [8]. In addition, we also analyzed HCK expression in primary MCLs alongside healthy B-cell subsets. Whereas HCK mRNA and protein were hardly detectable in naïve, GC and memory B-cells, or in plasmablasts (Fig. 1d \& Supplementary Fig. 1), high expression of HCK mRNA (Fig. 1a, Supplementary Fig. 1) and protein (Fig. 1d) was observed in 6 out of 7 primary MCLs, at similar levels as in Granta-519 (Fig. 1d). Taken together, HCK is aberrantly upregulated in MCL, high HCK expression correlates with poor patient survival, and enhanced HCK expression in B-cell malignancies is not dependent upon oncogenic MYD88 mutations.

To investigate whether HCK expression depends on (wild type) MYD88, we transduced JeKo-1 with doxycycline-inducible MYD88 shRNAs. MYD88 knockdown strongly reduced HCK protein and RNA levels, indicating that MYD88-mediated signaling drives HCK transcription in JeKo-1 (Fig. 1e, Supplementary Fig. 2). In contrast, underlining the specificity of this effect, expression of the Src family kinases Lyn, Lck, and Fyn was not affected (Fig. 1e and data not shown). Notably, HCK expression was not reduced upon serum deprivation of the cells, indicating a cell intrinsic mechanism for MYD88dependent HCK expression (data not shown). Since MYD88 plays a prominent role in TLR-signaling and TLR agonists from necrotic cells are abundantly present in the tumor microenvironment, we stimulated MCL cells with TLR agonists and analyzed HCK levels. Stimulation of JeKo-1, Granta-519, Maver-1, Mino, and primary MCL with TLR agonists enhanced $H C K$ mRNA (Supplementary Fig. 3) and HCK protein levels (Fig. 1f), in a MYD88dependent manner (Fig. 1g). Hence, HCK expression in MCL depends on constitutive MYD88 signaling and can be further enhanced by TLR stimulation. In line with these findings, GSEA of MCL patients with high versus low HCK expression showed enrichment of the KEGG TLR signaling pathway gene set (Supplementary Table 1 and Supplementary Fig. 4).

Since HCK has been identified as a pro-survival kinase in MYD88-mutated ABC-DLBCL and WM cell lines [8], we investigated if HCK is involved in MCL cell survival. JeKo-1 and Granta-519 were transduced with doxycyclineinducible HCK shRNAs and in both cell lines knockdown of HCK resulted in a significant decrease in the number of viable cells (Fig. 2a, b). To discriminate between effects on cell proliferation and apoptosis, we performed BrdU cell proliferation and Annexin- $\mathrm{V}$ apoptosis assays following HCK knockdown. In both cell lines HCK knockdown 
A

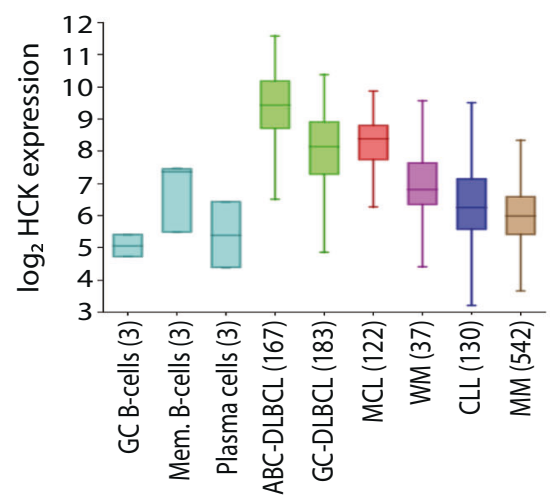

B

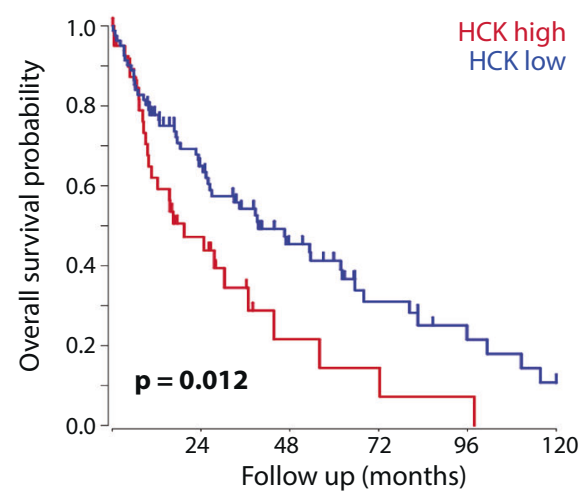

D

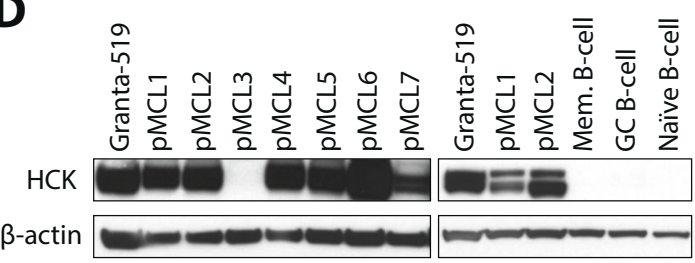

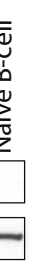

E

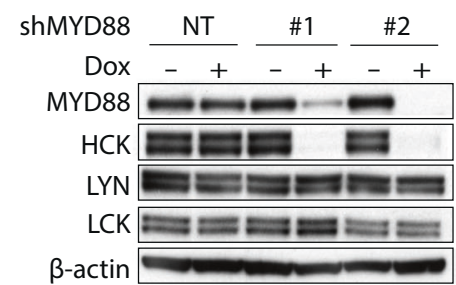

G

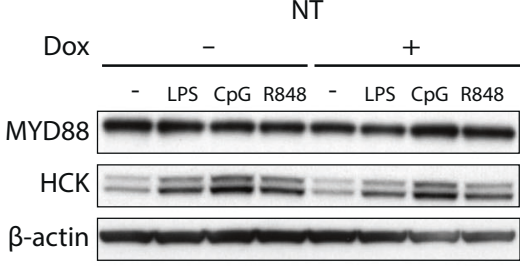

F

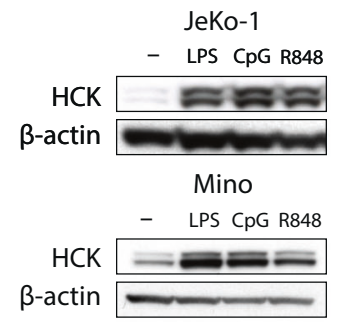

shMYD88 \#1

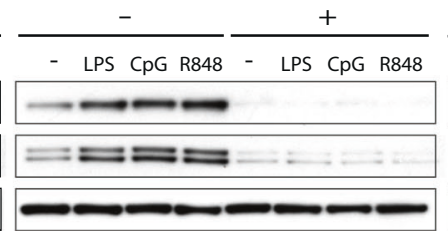

Granta-519

- LPS CPG R848

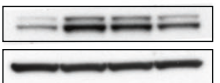

PMCL1

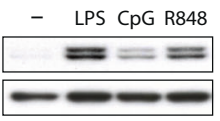

Maver-1
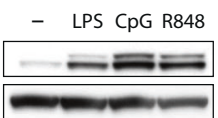

pMCL2

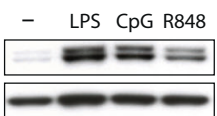




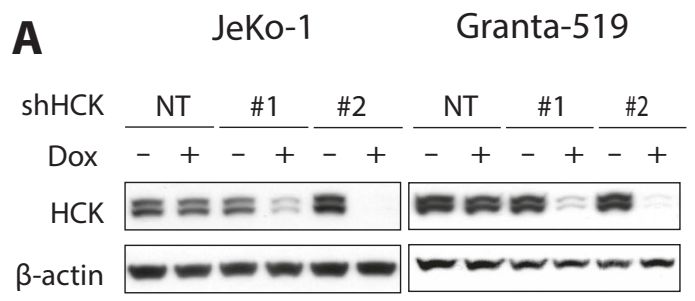

C JeKo-1

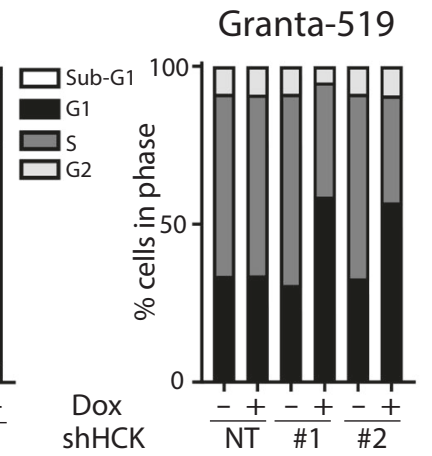

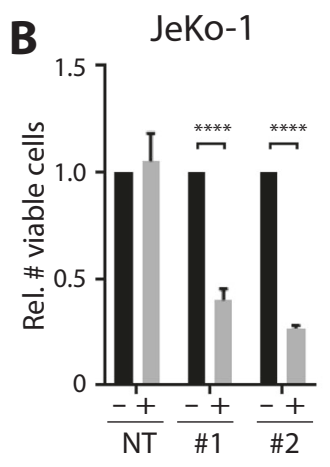

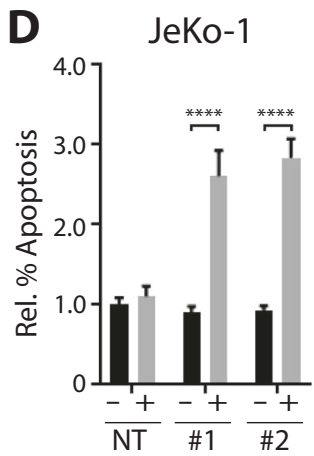

Granta-519

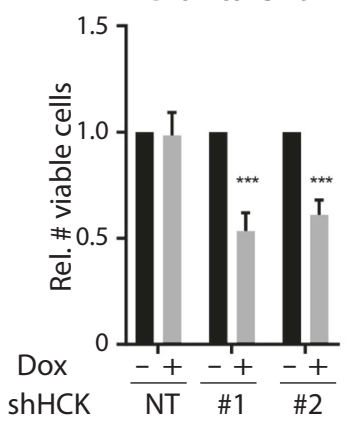

\section{Granta-519}

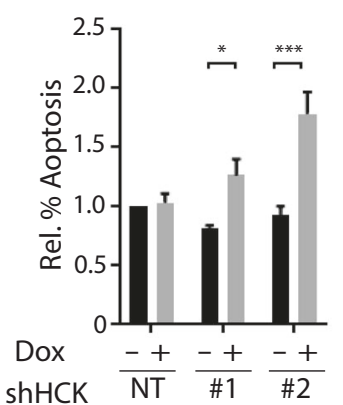

$\mathbf{E}$

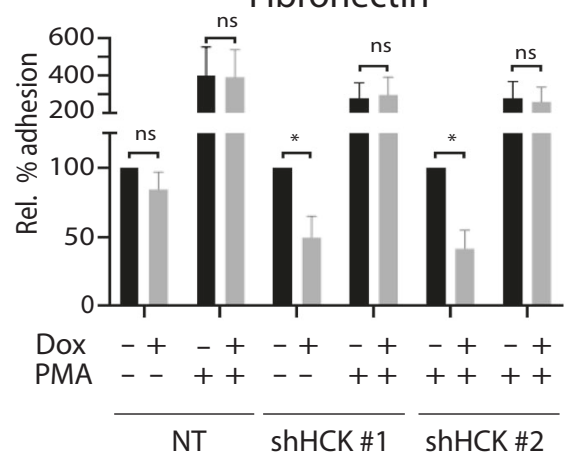

Fig. 2 HCK is critical for proliferation, survival and integrinmediated adhesion of MCL cells. a-f JeKo- 1 and Granta- 519 cells were transduced with pLKO-TET-puro plasmids encoding two shRNA's targeting HCK or a scrambled shRNA (NT). a HCK protein expression in MCL cell lines after 3 days of doxycycline treatment. Immunoblot, $\beta$-actin was used as a loading control. b Number of viable cells after HCK knockdown, determined by flow cytometric analysis and 7-AAD staining after 7 days of doxycycline treatment. Number of viable cells was normalized to the untreated condition. Data are presented as mean \pm S.E.M. of four independent experiments performed in triplicate. c Cell cycle analysis after HCK knockdown. The percentage of cells in Sub-G1 (BrdU-, <To-Pro- $\left.3^{-}\right)$, G1 (BrdU ${ }^{-}, \mathrm{To}^{-}$ Pro- $\left.3^{-}\right), \mathrm{S}\left(\mathrm{BrdU}^{+}\right)$, and $\mathrm{G} 2\left(\mathrm{BrdU}^{+}\right.$, To-Pro- $\left.3^{+}\right)$were determined by flow cytometric analysis after 7 days of doxycycline treatment. The graphs are representative for 3 individual experiments. d Apoptosis after HCK knockdown, defined as the percentage of Annexin- $\mathrm{V}$ positive cells after 7 days of doxycycline treatment. Percentage of Annexin- $\mathrm{V}$ positive cells were normalized to the untreated condition. Data are presented as mean \pm S.E.M. of three independent experiments. e Integrin-mediated adhesion of JeKo-1 cells after HCK knockdown. Cells were treated with doxycycline for 4 days and
HS-27a

G 
increased the percentage of cells in G1 and decreased the percentage of cells in S-phase (Fig. 2c). Furthermore, an increase in the amount of Annexin- $\mathrm{V}$ positive cells was observed after HCK knockdown (Fig. 2d). These data demonstrate that HCK regulates proliferation, i.e., G1/S transition, and survival of MCL cells.

As illustrated by the molecular mechanism underlying the clinical efficacy of the BTK inhibitor ibrutinib in MCL patients, MCL cells are critically dependent upon the interaction with their tumor microenvironment for in vivo survival and growth, and integrin-mediated adhesion is crucial for retention of the MCL cells in their protective lymphoid organ niche [12-14]. Interestingly, the integrin-mediated adhesion of MCL cells to fibronectin and the bone marrow stromal cell line HS-27a was significantly impaired after HCK knockdown (Fig. 2e, f). Notably, integrin activation by stimulation of PKC with PMA rescued this effect (Fig. 2e), demonstrating that the impaired adhesion upon HCK silencing does not reflect reduced cell viability or integrin expression (Supplementary Fig. 5a) and that PKC controls integrin activity independent or downstream of HCK. Pretreatment of MCL cells with the integrin $\alpha 4$ blocking antibody HP2/1 completely abrogated the adhesion to fibronectin and diminished the adhesion to the bone marrow stromal cell line HS-27a by more than $85 \%$ (Supplementary Fig. 5b), demonstrating the involvement of integrin $\alpha 4 \beta 1$, also in the interaction with stromal cells. Finally, to assess a possible role for HCK in adhesion of primary MCL, we employed a potent inhibitor of HCK, the pan-Src family kinase inhibitor A419259 [15]. Both primary MCL tested, as well as JeKo-1 cells, showed a strong dose-dependent reduction in the adhesion to fibronectin upon treatment with A419259 (Fig. 2g \& Supplementary Fig. 5c), and stimulation of PKC with PMA rescued this effect (Supplementary Fig. 5c). Although inhibition of other SFKs may also contribute to the observed reduction in adhesion, these data support a potential involvement of HCK in regulation of integrin-mediated adhesion of primary MCL cells as well.

Taken together, we demonstrate that HCK is aberrantly upregulated in MCL cell lines and primary patient samples, and high expression of HCK correlates with inferior prognosis. In MCL, HCK expression is MYD88-dependent, but not driven by mutant MYD88. Furthermore, TLR/MYD88signaling transcriptionally regulates HCK levels. Thus, the aberrant HCK expression in primary MCL cells may reflect local TLR stimulation in the tumor microenvironment. This notion is supported by the GSEA, showing enrichment of a TLR signaling pathway gene set in MCL patients with high expression of HCK. Functionally, HCK knockdown results in G1/S arrest and impaired cell viability. In addition, we established that HCK regulates integrin-mediated adhesion of MCL cells to the extracellular matrix and stromal cells. By analogy to our studies on the mechanism of action underlying the clinical efficacy of ibrutinib [12-14], this implies that
HCK inhibition could mobilize MCL cells from their protective lymphoid organ niche into the circulation, thereby depriving them from critical growth- and survival-factors. Combined with the restricted expression of HCK in hematopoietic cells, enhancing the likelihood of clinical safety, our results provide a strong rationale for clinical studies exploring the efficacy of targeted therapy with HCK inhibitors in MCL.

Acknowledgements This work is supported by grants from Lymph\&Co, the IWMF, and the Dutch Cancer Society (KWF).

Author contributions HCL designed the research, performed experiments, analyzed the data, designed the figures, and wrote the paper; $\mathrm{MM}$ and AK performed experiments; MK supervised the study and provided primary patient material; STP and MS supervised the study, designed the research, analyzed the data, and wrote the paper.

\section{Compliance with ethical standards}

Conflict of interest The authors declare that they have no conflict of interest.

Publisher's note Springer Nature remains neutral with regard to jurisdictional claims in published maps and institutional affiliations.

Open Access This article is licensed under a Creative Commons Attribution 4.0 International License, which permits use, sharing, adaptation, distribution and reproduction in any medium or format, as long as you give appropriate credit to the original author(s) and the source, provide a link to the Creative Commons license, and indicate if changes were made. The images or other third party material in this article are included in the article's Creative Commons license, unless indicated otherwise in a credit line to the material. If material is not included in the article's Creative Commons license and your intended use is not permitted by statutory regulation or exceeds the permitted use, you will need to obtain permission directly from the copyright holder. To view a copy of this license, visit http://creativecommons. org/licenses/by/4.0/.

\section{References}

1. Martin P, Maddocks K, Leonard JP, Ruan J, Goy A, WagnerJohnston N, et al. Postibrutinib outcomes in patients with mantle cell lymphoma. Blood. 2016;127:1559-63.

2. Ziegler SF, Marth JD, Lewis DB, Perlmutter RM. Novel proteintyrosine kinase gene (hck) preferentially expressed in cells of hematopoietic origin. Mol Cell Biol. 1987;7:2276-85.

3. El-Shazly A, Yamaguchi N, Masuyama K, Suda T, Ishikawa T. Novel association of the Src family kinases, Hck and c-Fgr, with CCR3 receptor stimulation: A possible mechanism for eotaxininduced human eosinophil chemotaxis. Biochem Bioph Res Co. 1999;264:163-70.

4. Scholz G, Cartledge K, Dunn AR. Hck enhances the adherence of lipopolysaccharide-stimulated macrophages via $\mathrm{Cbl}$ and phosphatidylinositol 3-kinase. J Biol Chem. 2000;275:14615-23.

5. Podar K, Mostoslavsky G, Sattler M, Tai YT, Hayashi T, Catley LP, et al. Critical role for hematopoietic cell kinase (Hck)-mediated phosphorylation of Gab1 and Gab2 docking proteins in interleukin 6-induced proliferation and survival of multiple myeloma cells. J Biol Chem. 2004;279:21658-65.

6. Kubo T, Kuroda Y, Shimizu H, Kokubu A, Okada N, Hosoda F, et al. Resequencing and copy number analysis of the human 
tyrosine kinase gene family in poorly differentiated gastric cancer. Carcinogenesis 2009;30:1857-64.

7. Roversi FM, Pericole FV, Machado-Neto JA, da Silva Santos Duarte A, Longhini AL, Corrocher FA, et al. Hematopoietic cell kinase (HCK) is a potential therapeutic target for dysplastic and leukemic cells due to integration of erythropoietin/PI3K pathway and regulation of erythropoiesis: HCK in erythropoietin/ PI3K pathway. Biochim Biophys Acta Mol Basis Dis. 2017; 1863:450-61.

8. Yang G, Buhrlage SJ, Tan L, Liu X, Chen J, Xu L, et al. HCK is a survival determinant transactivated by mutated MYD88, and a direct target of ibrutinib. Blood. 2016;127:3237-52.

9. Akhter A, Street L, Ghosh S, Burns BF, Elyamany G, ShabaniRad MT, et al. Concomitant high expression of Toll-like receptor (TLR) and B-cell receptor (BCR) signalling molecules has clinical implications in mantle cell lymphoma. Hematol Oncol. 2017;35:79-86.

10. Wang L, Zhao Y, Qian J, Sun L, Lu Y, Li H, et al. Toll-like receptor-4 signaling in mantle cell lymphoma: effects on tumor growth and immune evasion. Cancer. 2013;119:782-91.
11. Mastorci K, Muraro E, Pasini E, Furlan C, Sigalotti L, Cinco M, et al. Toll-like receptor $1 / 2$ and 5 ligands enhance the expression of cyclin D1 and D3 and induce proliferation in mantle cell lymphoma. PLoS One 2016;11:e0153823.

12. Chang BY, Francesco M, De Rooij MF, Magadala P, Steggerda SM, Huang MM, et al. Egress of CD19(+)CD5(+) cells into peripheral blood following treatment with the Bruton tyrosine kinase inhibitor ibrutinib in mantle cell lymphoma patients. Blood. 2013;122:2412-24.

13. de Rooij MF, Kuil A, Kater AP, Kersten MJ, Pals ST, Spaargaren M. Ibrutinib and idelalisib synergistically target BCR-controlled adhesion in MCL and CLL: a rationale for combination therapy. Blood. 2015;125:2306-9.

14. de Rooij MF, Kuil A, Geest CR, Eldering E, Chang BY, Buggy JJ, et al. The clinically active BTK inhibitor PCI-32765 targets Bcell receptor- and chemokine-controlled adhesion and migration in chronic lymphocytic leukemia. Blood. 2012;119:2590-4.

15. Saito Y, Yuki H, Kuratani M, Hashizume Y, Takagi S, Honma T, et al. A pyrrolo-pyrimidine derivative targets human primary AML stem cells in vivo. Sci Transl Med. 2013;5:181ra52. 